\title{
Complex Analysis of the Stellar Binary HD25811: A Subgiant System
}

\author{
Mashhoor A. Al-Wardat ${ }^{1,3}$, Hatem S. Widyan ${ }^{2}$ and Ahmed Al-thyabat ${ }^{2}$ \\ ${ }^{1}$ Department of Physics, Al-Hussein Bin Talal University, P.O. Box 20, 71111, Ma'an, Jordan \\ ${ }^{2}$ Department of Physics, Al al-Bayt University, Mafraq, Jordan \\ ${ }^{3}$ Email: mwardat@ahu.edu.jo
}

(ReCEIVED June 21, 2013; AcCEPTED November 19, 2013)

\begin{abstract}
The visually close binary system HD25811 is analysed to estimate its physical and geometrical parameters in addition to its spectral type and luminosity class. The method depends on obtaining the best fit between the entire observational spectral energy distribution (SED) of the system and synthetic SEDs created by atmospheric modelling of the individual components, consistent with the system's modified orbital elements. The parameters of the individual components of the system are derived as: $T_{\text {eff }}^{\mathrm{a}}=6850 \pm 50 \mathrm{~K}, T_{\text {eff }}^{\mathrm{b}}=7000 \pm 50 \mathrm{~K}, \log g_{\mathrm{a}}=4.04 \pm 0.10, \log g_{\mathrm{b}}=4.15 \pm 0.10, R_{\mathrm{a}}=$ $1.96 \pm 0.20 \mathrm{R}_{\odot}, R_{\mathrm{b}}=1.69 \pm 0.20 \mathrm{R}_{\odot}, M_{v}^{\mathrm{a}}=1 .{ }^{\mathrm{m}} 97 \pm 0.20, M_{v}^{\mathrm{b}}=2{ }^{\mathrm{m}} 19 \pm 0.20, L_{a}=7.59 \pm 0.70 L_{\odot}, L_{b}=$ $6.16 \pm 0.70 L_{\odot}$ with dynamical parallax $\pi$ (mas) $=5.095 \pm 0.095$. The analysis shows that the system consists of a $1.55 M_{\odot}$ F2 subgiant star and a less evolved $1.50 M_{\odot}$ F1 secondary subgiant star with ages around 2 Gy formed by fragmentation. Synthetic magnitudes of both components were calculated under Johnson-Cousins, Strömgren, and Tycho photometrical systems.
\end{abstract}

Keywords: stars: fundamental parameters - binaries: close - stars: distances - stars: formation - stars: individual: HD25811

\section{INTRODUCTION}

Analysis of stellar binary systems is the most reliable and accurate way of estimating stellar physical and geometrical parameters, especially stellar masses. It emphasises the role of binary stars in examining some physical and stellar evolutionary theories. Even high resolution observational techniques, like speckle interferometry and adaptive optics, are not sufficient to determine the physical parameters of the individual components of visually close (spatially unresolved on the sky) binary systems (VCBS), especially in the case of this study (HD25811) which has no parallax measurement.

Combining spectrophotometry with atmospheric modelling gives a new method for the accurate determination of the physical and geometrical parameters of both components of VCBS, in addition to the estimation of their spectral types, luminosity classes, and ages. This method, first advised by Al-Wardat (2002a, 2007), was applied to several VCBS such as ADS11061, Cou1289, Cou1291, Hip11352, Hip11253, Hip70973, and Hip72479 (Al-Wardat 2002a, 2007, 2009, 2012; Al-Wardat \& Widyan 2009).
In this paper, we are developing the method by combining the dynamical analysis of the relative orbit of the binary, a step which will strengthen the method, reduce the error bars, and make it applicable to evolved binaries by accurate determination of the individual masses.

The system HD25811 (SAO $93759=$ CHA13 = BAG 4) was first resolved as a binary using the lunar-occultation observational technique in the early eighties (Schmidtke \& Africano 1984; Evans et al. 1985). Subsequently, it was included in the speckle interferometric programme of the Russian 6-metre telescope by Balega \& Balega (1987), and was designated as BAG 4. Since then it is routinely observed by speckle interferometric techniques all over the world to achieve as much data as possible in order to calculate its best orbit (McAlister et al. 1987; Balega, Balega, \& Vasyuk 1989; McAlister et al. 1989, 1990; Hartkopf, McAlister, \& Franz 1992; Bagnuolo et al. 1992; Balega et al. 1994, 2001, 2007)(see Table 1). But it was not listed in the Hipparcos programme, which is why it has no trigonometric parallax measurement.

The VCBS HD25811 (BAG 4) fulfils the requirements of the aforementioned method, where it has precise magnitude 
Table 1. Relative positional measurements using different methods which are used to build the orbit of the system. These points are taken from the Fourth Catalog of Interferometric Measurements of Binary Stars and a point from Al-Wardat (2003).

\begin{tabular}{lclcc}
\hline \hline Date & $\theta(\mathrm{deg})$ & $\rho(\mathrm{deg})$ & Ref. & Meth. \\
\hline 1984.1127 & 66.7 & 0.0701 & Smk1984 & Occ \\
1984.1127 & 254.4 & 0.0653 & Evn1985 & Occ \\
1984.1127 & 254.4 & 0.0663 & Evn1985 & Occ \\
1984.8460 & 81.0 & 0.066 & Bag1987 & Spe \\
1985.8406 & 65.4 & 0.075 & McA1987b & Sch \\
1986.6573 & 64.8 & 0.072 & Bag1989a & Spe \\
1986.8862 & 59.9 & 0.074 & McA1989 & Sch \\
1986.8890 & 60.6 & 0.079 & McA1989 & Sch \\
1987.7655 & 56.9 & 0.074 & McA1989 & Sch \\
1988.6609 & 52.2 & 0.073 & McA1990 & Sch \\
1989.7067 & 41.5 & 0.076 & Hrt1992b & Sch \\
1989.8077 & 37.7 & 0.068 & Bag1994 & Spe \\
1990.7551 & 21.9 & 0.078 & Hrt1992b & Sch \\
1993.8419 & 353.0 & 0.056 & Bag1994 & Spe \\
1997.808 & 276.9 & 0.0582 & Bag2001 & Spe \\
1998.7747 & 266.9 & 0.060 & Bag2002 & Spe \\
1999.8185 & 259.4 & 0.0681 & Bag2004 & Spe \\
1999.8213 & 258.9 & 0.0683 & Bag2004 & Spe \\
2001.7614 & 247.3 & 0.076 & Bag2006b & Spe \\
2001.7614 & 246.9 & 0.076 & Bag2006b & Spe \\
2002.797 & 240.78 & 0.0880 & Wrd2003 & Spe \\
2004.8158 & 229.0 & 0.074 & Bag2007b & Spe \\
\hline \hline
\end{tabular}

Note: Occ $=$ occultaion; Spe $=$ speckle interferometry; Sch $=$ CHARA speckle; Wrd2003 = Al-Wardat (2003). References are abbreviated as in the Fourth Catalog of Interferometric Measurements of Binary Stars.

difference measurements and observational spectral energy distribution (SED) covers the optical range. It has also several relative positional measurements listed in the Fourth Catalog of Interferometric Measurements of Binary Stars (http://ad.usno.navy. mil/wds/int4.html), which they used to build its preliminary orbit and a new positional measurement that can be used to modify it.

In addition to that, HD26811 represents a very good example for studying the formation and evolution of stellar binary systems, since, as we shall prove, it consists of two subgiant stars.

Table 2 contains the basic data of the system from SIMBAD and other databases, and Table 3 contains data from Tycho-2 Catalogue (ESA 1997).
Table 2. Data from SIMBAD and NASA/IPAC.

\begin{tabular}{lcc}
\hline \hline & HD25811 & Source \\
\hline$\alpha_{2000}$ & $04^{h} 06^{m} 16 .^{\mathrm{s}} 416$ & 1 \\
$\delta_{2000}$ & $+19^{\circ} 52^{\prime} 28 .^{\prime \prime} 57$ & 1 \\
Tyc & $1258-22-1$ & 1 \\
HD & 25811 & 1 \\
Sp. Typ. & $\mathrm{F} 0$ & 1 \\
E(B-V) & 0.278 & 2 \\
$A_{v}$ & $0 .^{\mathrm{m}} 870$ & 2 \\
\hline \hline
\end{tabular}

Note: $1=$ SIMBAD; 2 = NASA/IPAC:http://irsa.ipac. caltech.edu

Table 3. Data from Tycho-2 catalogue (Høg et al. 2000).

\begin{tabular}{lc}
\hline \hline & $\mathrm{HD} 25811$ \\
\hline$V_{J}($ Hip $)$ & $8 .^{\mathrm{m}} 66$ \\
$B_{T}$ & $9 .{ }^{\mathrm{m}} 11 \pm 0.020$ \\
$V_{T}$ & $8 .^{\mathrm{m}} 71 \pm 0.017$ \\
$(B-V)_{J}(T y c)$ & $0 .{ }^{\mathrm{m}} 389 \pm 0.028$ \\
\hline \hline
\end{tabular}

\section{ORBITAL ELEMENTS}

The orbit of the system was calculated firstly by Balega et al. (2001) using the first 15 relative positional measurements in Table 1, they noted that they will have to wait another $\sim 10$ years to obtain a reliable solution. Then it was modified by Al-Wardat (2003) using the measurements up to 2002.797. A new slight modification of the orbit is introduced here using all relative positional measurements listed in Table 1, which cover around $210^{\circ}$ of the complete orbit.

The estimated orbital parameters of the system along with those of the old orbits are listed in Table 4.

Figure 1 shows the relative visual orbit of the system with the epoch of the positional measurements, and Figure 2 shows the new orbit against the old orbits of Balega et al. (2001) and Al-Wardat (2003).

Unfortunately, there is no any positional measurement for the system during the last 9 years, which would have covered the whole orbital period. This could give a more reliable orbit and hence more precise geometrical parameters.

Table 4. Estimated orbital elements of the system with those of the old orbits by Balega et al. (2001) and Al-Wardat (2003).

\begin{tabular}{lccc}
\hline \hline Parameters & This work & Al-Wardat (2003) & Balega et al. (2001) \\
\hline Period $P$ & $32.99^{y} \pm 2.37^{y}$ & $31.00^{y} \pm 1.33^{y}$ & $30.68^{y}$ \\
Periastron epoch $T_{0}$ & $1993.26 \pm 18.76$ & $1981 \pm 12$ & 1990.96 \\
Eccentricity $e$ & $0.047 \pm 0.040$ & $0.007 \pm 0.032$ & 0.045 \\
Semi-major axis $a$ & $0 .^{\prime \prime} 076 \pm 0 .^{\prime \prime} 002$ & $0 .^{\prime \prime} 076 \pm 0 .^{\prime \prime} 001$ & $0 .^{\prime \prime} 079$ \\
Inclination $i$ & $123^{\circ} \pm 3^{\circ}$ & $124^{\circ} \pm 2^{\circ}$ & $128^{\circ}$ \\
Argument of periastron $\omega$ & $68^{\circ} \pm 216^{\circ}$ & $291^{\circ} \pm 140^{\circ}$ & $32^{\circ}$ \\
Position angle of nodes $\Omega$ & $59^{\circ} \pm 3^{\circ}$ & $61^{\circ} \pm 1^{\circ}$ & $50^{\circ}$ \\
\hline \hline
\end{tabular}




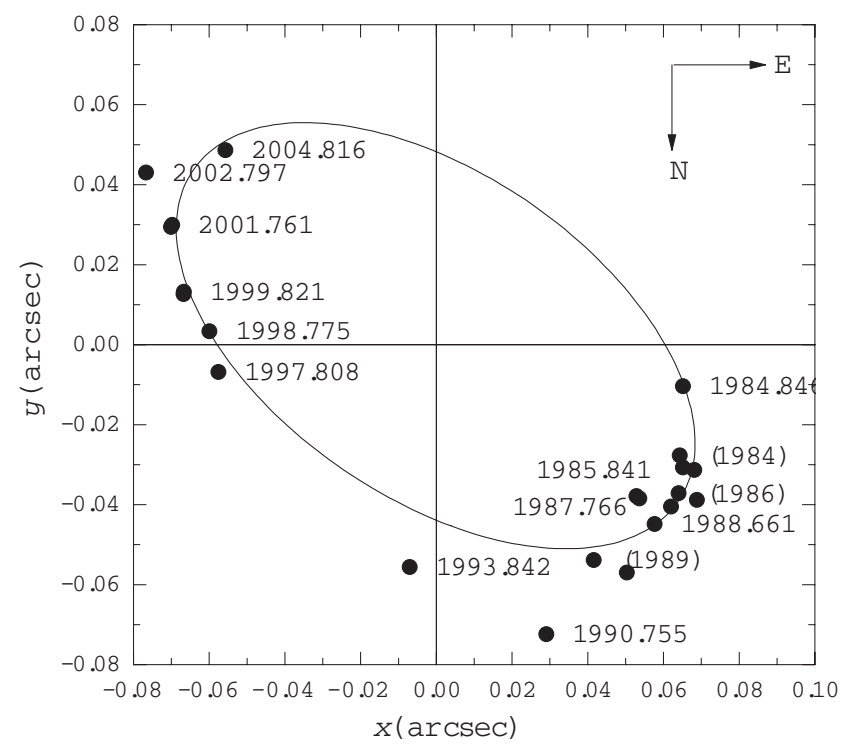

Figure 1. Relative visual orbit of the system with the epoch of the positional measurements; the origin represents the position of the primary component.

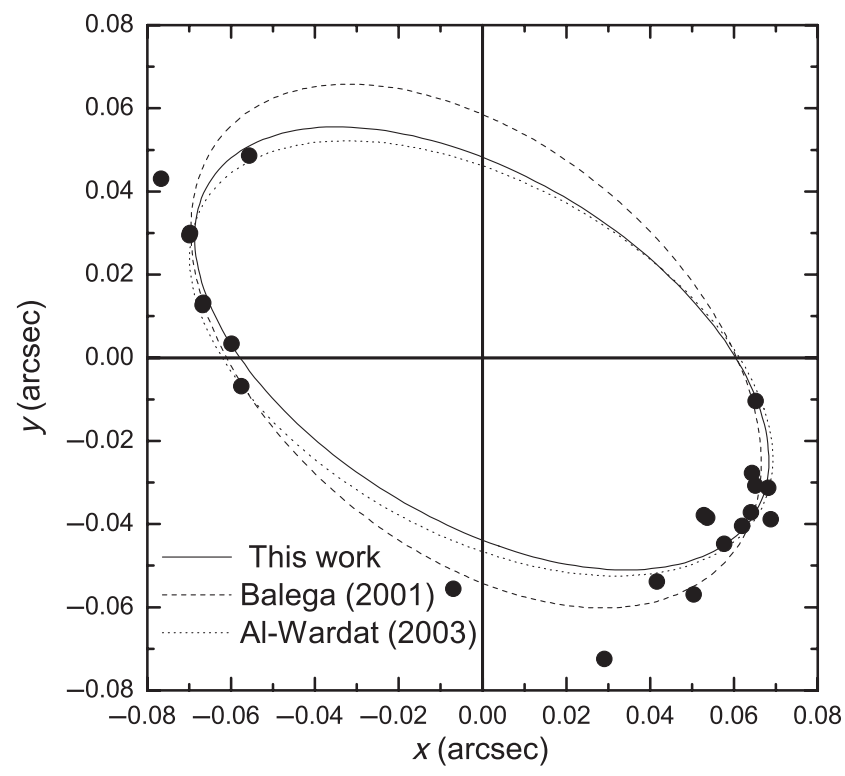

Figure 2. Comparison between the modified relative visual orbit of the system in this work (solid line) and those of Balega et al. (2001) (dashed line) and Al-Wardat (2003) (doted line).

\section{ATMOSPHERIC MODELLING}

To calculate the individual components' preliminary input parameters ( $T_{\text {eff }}$ and $\log g$ ), we followed the procedures explained in Al-Wardat (2012) using the following relations (e.g., Lang 1992; Gray 2005):

$$
\begin{gathered}
m_{v}=-2.5 \log \left(f_{1}+f_{2}\right) \\
M_{v}=m_{v}+5-5 \log (d)-A, \\
\log \left(R / R_{\odot}\right)=0.5 \log \left(L / L_{\odot}\right)-2 \log \left(T / T_{\odot}\right), \\
\log g=\log \left(M / M_{\odot}\right)-2 \log \left(R / R_{\odot}\right)+4.43,
\end{gathered}
$$

Table 5. Magnitude difference between the components of the system, along with the filter used to obtain the observations.

\begin{tabular}{lcr}
\hline \hline$\Delta m$ & Filter $(\lambda / \Delta \lambda)$ & Ref. \\
\hline $0 .{ }^{\mathrm{m}} 00 \pm 0.40$ & $545 \mathrm{~nm} / 30$ & 1 \\
$0 .{ }^{\mathrm{m}} 18 \pm 0.12$ & $610 \mathrm{~nm} / 20$ & 2 \\
$0 .{ }^{\mathrm{m}} 23 \pm 0.27$ & $545 \mathrm{~nm} / 30$ & 2 \\
$0 .{ }^{\mathrm{m}} 22 \pm 0.20$ & $850 \mathrm{~nm} / 75$ & 3 \\
$0 .{ }^{\mathrm{m}} 21 \pm 0.17$ & $545 \mathrm{~nm} / 30$ & 3 \\
$0 .{ }^{\mathrm{m}} 24 \pm 0.27$ & $545 \mathrm{~nm} / 30$ & 4 \\
\hline \hline
\end{tabular}

Note: 1 = Balega et al. (2002); 2 = Balega et al. (2004); 3 = Balega et al. (2006); $4=$ Balega et al. (2007).

where we used the bolometric corrections of Lang (1992) $\&$ Gray (2005), $T_{\odot}=5777 \mathrm{~K}$ and extinction $\left(A_{v}\right)$ given in Table 1 by NASA/ IPAC.

So, using the entire visual magnitude of the system $m_{v}=$ $8 .{ }^{\mathrm{m}} 66$ from Table 3 and $\Delta m=0 .{ }^{\mathrm{m}} 23$ from speckle interferometric results (Table 5) as the average of $\Delta m$ measurement under the filter $545 \mathrm{~nm} / 30$ (the closest filter to the Johnson V filter), we got $m_{v \mathrm{a}}=9^{n} \cdot 304$ and $m_{v \mathrm{~b}}=9^{n} \cdot 534$.

The dynamical parallax estimated by Al-Wardat (2003) $(\pi=5.24 \pm 0.6, d=191 \mathrm{pc})$ is used as a preliminary value to calculate the individual absolute magnitudes according to the following equation:

$$
M_{v}=m_{v}+5-5 \log (d)-A .
$$

This leads to the following preliminary input parameters:

$$
\begin{array}{cc}
M_{v \mathrm{a}}=2^{m} \cdot 03, & M_{v \mathrm{~b}}=2^{m} \cdot 26 \\
T_{\text {eff }}^{\mathrm{a}}=8150 \mathrm{~K}, & T_{\text {eff }}^{\mathrm{b}}=7950 \mathrm{~K}, \\
\log g_{\mathrm{a}}=4.2, & \log g_{\mathrm{b}}=4.2,
\end{array}
$$

and Spectral Types A6, A7. Which in their turn used to build the preliminary line blanketed model atmospheres using ATLAS 9 (Kurucz 1994).

\subsection{Synthetic spectra}

The observational SED of the entire system was taken from Al-Wardat (2002b) (Figure 3). It shows some strong lines and depressions, especially in the red part of the spectrum (around $\lambda 6867 \AA, \lambda 7200 \AA$, and $\lambda 7605 \AA$ ). These are $\mathrm{H}_{2} \mathrm{O}$ and $\mathrm{O}_{2}$ telluric lines and depressions. Synthetic Jonson-Cousins, Strömgren, and Tycho magnitudes and colour indices of this observational SED are listed in Table 6.

Synthetic SEDs of the individual components are built first using solar metalicity model atmospheres as the output of ATLAS 9 of the preliminary parameters (Equs. 6, 7, and 8), $R_{\mathrm{a}}=1.55 \mathrm{R}_{\odot}, R_{\mathrm{b}}=1.45 \mathrm{R}_{\odot}$, and $d=191 \mathrm{pc}$ (from Al-Wardat 2003). The total energy flux from a binary star is created from the net luminosities of the components $a$ and $b$ located at a distance $d$ from the Earth as follows:

$$
F_{\lambda} \cdot d^{2}=H_{\lambda}^{\mathrm{a}} \cdot R_{\mathrm{a}}^{2}+H_{\lambda}^{\mathrm{b}} \cdot R_{\mathrm{b}}^{2},
$$


Table 6. Entire synthetic Johnson-Cousins, Strömgren, and Tycho magnitudes and colour indices of the system HD25811 (Al-Wardat 2008).

\begin{tabular}{lcc}
\hline \hline System & Filter & HD25811 \\
\hline Johnson-Cousins & $B_{J}$ & $9 .{ }^{\mathrm{m}} 05$ \\
& $V_{J}$ & $8 .^{\mathrm{m}} 64$ \\
Strömgren & $(B-V)_{J}$ & $0 .{ }^{\mathrm{m}} 41$ \\
& $v$ & $9 .{ }^{\mathrm{m}} 31$ \\
& $b$ & $8 .^{\mathrm{m}} 91$ \\
& $y$ & $8 .{ }^{\mathrm{m}} 61$ \\
Tycho & $v-b$ & $0 .{ }^{\mathrm{m}} 40$ \\
& $b-y$ & $0 .{ }^{\mathrm{m}} 30$ \\
& $B_{T}$ & $9 .{ }^{\mathrm{m}} 14$ \\
& $V_{T}$ & $8 .{ }^{\mathrm{m}} 70$ \\
\hline \hline
\end{tabular}

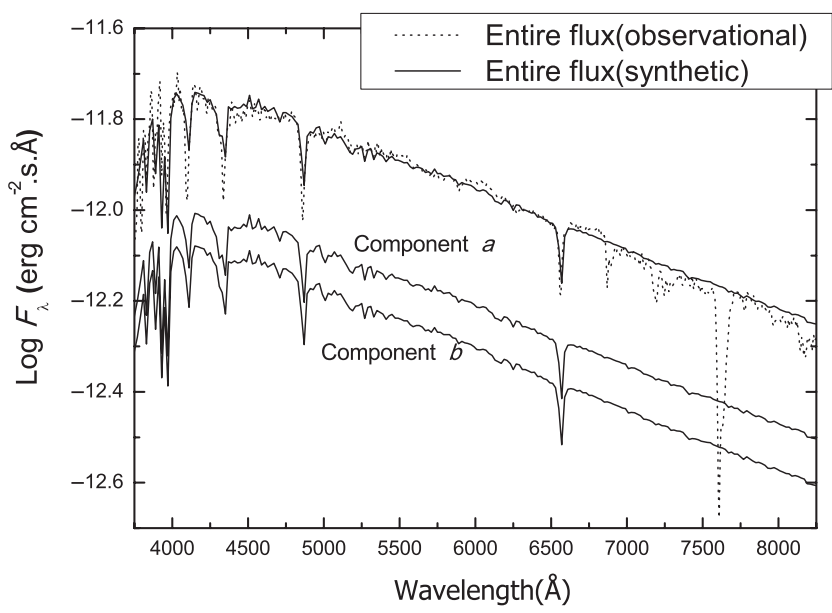

Figure 3. Dotted line: observational SED in the continuous spectrum of the system. Solid lines: the entire computed SED of the two components, the computed flux of the primary component with $T_{\text {eff }}=6850 \pm 50 \mathrm{~K}, \log g=$ $4.04 \pm 0.10, R=1.96 \pm 0.20 R_{\odot}$, and the computed flux of the secondary component with $T_{\text {eff }}=7000 \pm 50 \mathrm{~K}, \log g=4.15 \pm 0.10, R=1.69 \pm$ $0.20 R_{\odot}$, and $d=196.27 \mathrm{pc}$.

from which

$$
F_{\lambda}=\left(R_{\mathrm{a}}^{2} / d\right)^{2}\left(H_{\lambda}^{\mathrm{a}}+H_{\lambda}^{\mathrm{b}} \cdot\left(R_{\mathrm{b}} / R_{\mathrm{a}}\right)^{2}\right),
$$

where $H_{\lambda}^{\mathrm{a}}$ and $H_{\lambda}^{\mathrm{b}}$ are the fluxes from a unit surface of the corresponding component. $F_{\lambda}$ here represents the entire SED of the system; which for a given $H_{\lambda}^{\mathrm{a}, \mathrm{b}}$ depends on the parallax of the system and the radii of the components.

The resultant entire synthetic SED did not fit the observational SED. Therefore, many attempts were made and hundreds of synthetic SEDs were built, using different sets of parameters, and compared with the observational SED to achieve the best fit. The attempts included changing the effective temperatures, gravity acceleration, parallax, and radii. It is worthwhile mentioning that the step size of the change was less than or equal to the estimated error in each parameter. In the case of the effective temperatures and radii, the changes exceed the errors of the first estimations because we are dealing with subgiants instead of main sequence, or in other words, that is what we discovered.

Kepler's equation is used to match between the physical and geometrical parameters, and to connect the dynamical analysis with the atmospheric modelling:

$$
\left(\frac{M_{a}+M_{b}}{M_{\odot}}\right)\left(\pi^{3}\right)=\frac{a^{3}}{p^{2}},
$$

where $\left(\frac{M_{a}+M_{b}}{M_{\odot}}\right)$ is the mass sum of the two components in terms of solar mass, $\pi$ is the parallax of the system in arc seconds, $a$ is the semi-major axes in arc seconds, and $p$ is the period in years.

Fixing the right part of Equation (11) according to the orbital elements of the system calculated in Section 2, and changing the values of the left part in an iterated way, ensures agreement between the masses of the components, their positions on the evolutionary tracks, and the best fit between the synthetic and observational SEDs.

For stars with formerly estimated temperatures, masses, and gravity accelerations, this agreement is possible only for radii bigger than what would be if they were main sequence stars (the preliminary input radii), which means that both components are evolved subgiant stars.

Within the criteria of the best fit, which are the maximum values of the absolute fluxes, the inclination of the continuum of the spectra, and the profiles of the absorption lines, the best fit (Figure 3) is found using the following set of parameters:

$$
\begin{gathered}
T_{\text {eff }}^{\mathrm{a}}=6850 \pm 50 \mathrm{~K}, T_{\text {eff }}^{\mathrm{b}}=7000 \pm 50 \mathrm{~K}, \\
\log g_{\mathrm{a}}=4.04 \pm 0.10, \log g_{\mathrm{b}}=4.15 \pm 0.10, \\
R_{\mathrm{a}}=1.96 \pm 0.20 \mathrm{R}_{\odot}, R_{\mathrm{b}}=1.69 \pm 0.20 \mathrm{R}_{\odot}, \\
\left(M_{a}+M_{b}\right) / M_{\odot}=3.05 \pm 0.10,
\end{gathered}
$$

and $5.095 \pm 0.095, d=196.27 \mathrm{pc}$.

The role of Equation (11) rises here to assure the compatibility between the parallax of the system and its mass sum being given the period and the semi-major axes.

The complete set of the physical and geometrical parameters are listed in Table 7.

The estimated parameters are highly dependent on the precision of observations. Thus, within the error values of the measured quantities, they represent adequately enough the parameters of the systems' components.

Depending on the tables of Straizys \& Kuriliene (1981) and Gray (2005), the spectral types for the components of the system are estimated as F2 IV for the primary component and F1 IV for the secondary component.

\section{SYNTHETIC PHOTOMETRY}

In addition to its importance in calculating the entire and individual synthetic magnitudes of the system, synthetic photometry is used here as an evaluation technique to examine the best fit between the synthetic and observational spectra. This is performed by comparing the observed magnitudes 
Table 7. Physical and geometrical parameters of the system HD25811 components.

\begin{tabular}{lcc}
\hline \hline Component & $a$ & $b$ \\
\hline$T_{\text {eff }}(\mathrm{K})$ & $6850 \pm 50$ & $7000 \pm 50$ \\
Radius $\left(\mathrm{R}_{\odot}\right)$ & $1.96 \pm 0.20$ & $1.69 \pm 0.20$ \\
$\log g$ & $4.04 \pm 0.10$ & $4.15 \pm 0.10$ \\
$L\left(L_{\odot}\right)$ & $7.59 \pm 0.70$ & $6.16 \pm 0.70$ \\
$M_{V}$ & $1 .^{\mathrm{m}} 97 \pm 0.20$ & $2 .^{\mathrm{m}} 19 \pm 0.20$ \\
Mass, $\left(M_{\odot}\right)$ & $1.55 \pm 0.16$ & $1.5 \pm 0.15$ \\
$\bar{\rho}\left(\bar{\rho}_{\odot}\right)$ & $0.21 \pm 0.05$ & $0.31 \pm 0.05$ \\
Sp. Type & $\mathrm{F} 2 \mathrm{IV}$ & $\mathrm{F} 1 \mathrm{IV}$ \\
Parallax $(\mathrm{mas})$ & \multicolumn{2}{c}{$5.095 \pm 0.095$} \\
$\left(M_{a}+M_{b}\right) / M_{\odot}$ & \multicolumn{2}{c}{$3.05 \pm 0.10$} \\
1 Age $(\mathrm{Gy})$ & \multicolumn{2}{c}{$2.0 \pm 0.4$} \\
\hline \hline
\end{tabular}

Table 8. Synthetic magnitudes and colour indices of the system.

\begin{tabular}{lcrrr}
\hline \hline $\begin{array}{l}\text { Photomet. } \\
\text { System }\end{array}$ & Filter & $\begin{array}{r}\text { Entire } \\
\pm 0.03\end{array}$ & $\begin{array}{c}\text { Comp. } \\
a\end{array}$ & $\begin{array}{c}\text { Comp. } \\
\text { Johnson- }\end{array}$ \\
Cousins & $U$ & 9.04 & 9.70 & 9.89 \\
& $B$ & 9.04 & 9.70 & 9.89 \\
& $V$ & 8.66 & 9.31 & 9.53 \\
& $R$ & 8.44 & 9.08 & 9.32 \\
& $U-B$ & 0.00 & 0.00 & 0.00 \\
& $B-V$ & 0.38 & 0.40 & 0.36 \\
Strömgren & $V-R$ & 0.22 & 0.23 & 0.21 \\
& $u$ & 10.25 & 10.91 & 11.11 \\
& $v$ & 9.30 & 9.96 & 10.14 \\
& $b$ & 8.88 & 9.54 & 9.74 \\
& $y$ & 8.64 & 9.28 & 9.51 \\
& $u-v$ & 0.96 & 0.95 & 0.97 \\
Tycho & $v-b$ & 0.42 & 0.43 & 0.40 \\
& $b-y$ & 0.25 & 0.25 & 0.24 \\
& $B_{T}$ & 9.13 & 9.79 & 9.98 \\
& $V_{T}$ & 8.71 & 9.36 & 9.58 \\
& $B_{T}-V_{T}$ & 0.42 & 0.44 & 0.40 \\
\hline \hline
\end{tabular}

of the entire system from different sources with the entire synthetic ones using the following relation Maíz Apellániz (2006) and Maíz Apellániz (2007):

$$
m_{p}\left[F_{\lambda, s}(\lambda)\right]=-2.5 \log \frac{\int P_{p}(\lambda) F_{\lambda, s}(\lambda) \lambda \mathrm{d} \lambda}{\int P_{p}(\lambda) F_{\lambda, r}(\lambda) \lambda \mathrm{d} \lambda}+\mathrm{ZP}_{p}
$$

where $m_{p}$ is the synthetic magnitude of the passband $p, P_{p}(\lambda)$ is the dimensionless sensitivity function of the passband $p$, $F_{\lambda, s}(\lambda)$ is the synthetic SED of the object, and $F_{\lambda, r}(\lambda)$ is the SED of the reference star (Vega). Zero points $\left(\mathrm{ZP}_{p}\right)$ from Maíz Apellániz (2007) (and references therein) were adopted.

Table 8 shows the magnitudes and colour indices of the entire system and individual components in $U B V R$ JohnsonCousins, $u v b y$ Strömgren, and $B V$ Tycho.
Table 9. Comparison between the observational and synthetic magnitudes, colours, and magnitude differences of the system.

\begin{tabular}{lcc}
\hline \hline & Obs. ${ }^{a}$ & Synth. (this work) \\
\hline$V_{J}$ & $8 .^{\mathrm{m}} 66$ & $8 .^{\mathrm{m}} 66 \pm 0.03$ \\
$B_{T}$ & $9 \cdot^{\mathrm{m}} 11 \pm 0.020$ & $9 .^{\mathrm{m}} 13 \pm 0.03$ \\
$V_{T}$ & $8 .^{\mathrm{m}} 71 \pm 0.017$ & $8 .^{\mathrm{m}} 71 \pm 0.03$ \\
$(B-V)_{J}$ & $0 .{ }^{\mathrm{m}} 389 \pm 0.028$ & $0 .^{\mathrm{m}} 38 \pm 0.04$ \\
$\Delta m$ & $0 .{ }^{\mathrm{m}} 23^{b}$ & $0 .^{\mathrm{m}} 22 \pm 0.04$ \\
\hline \hline
\end{tabular}

${ }^{a}$ See Table 2.

${ }^{b}$ Average value of the observational measurements using the filter $545 \mathrm{~nm} / 30$ (Table 5) .

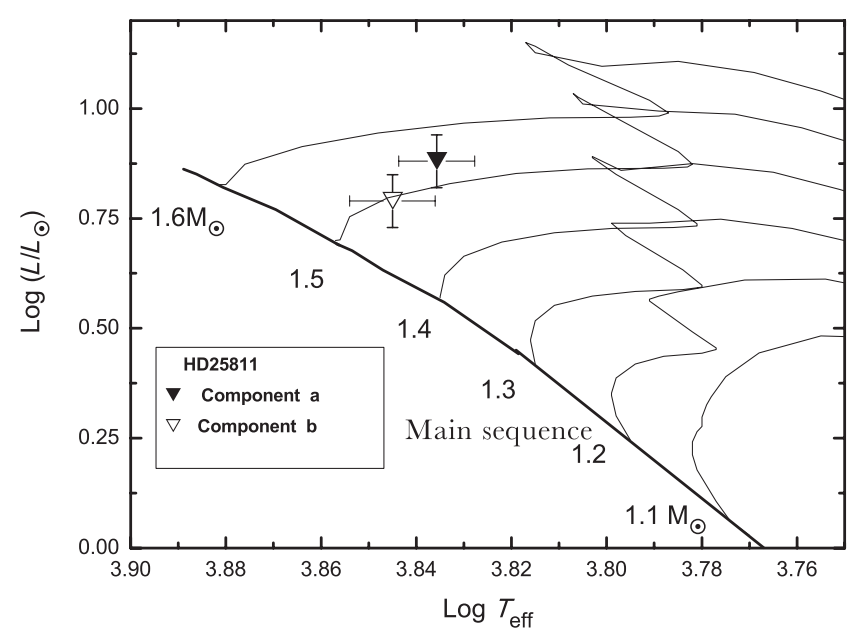

Figure 4. Components of the system on the evolutionary tracks of Girardi et al. (2000).

\section{RESULTS AND DISCUSSION}

Comparing the synthetic magnitudes and colours with the observational ones (Table 9) shows a high consistency between them. This gives a good indication about the reliability of the estimated parameters of the individual components of the system which are listed in Table 7.

Figure 4 shows the positions of the system's components on the evolutionary tracks of Girardi et al. (2000). It shows that the primary more massive component is more evolved than the secondary, which is why the estimated value of the luminosity of the primary is greater than the expected value for an MS star of the same mass.

The estimated parameters of the system (Table 7) show that the components of the system are similar to the star $\beta$ Hydri (HIP 2021), which is a G2IV evolved subgiant with an age of about 6.5-7.0 Gyr (Dravins, Lindegren, \& Vandenberg 1998; Fernandes \& Monteiro 2003), $\bar{\rho}\left(\bar{\rho}_{\odot}\right)=0.1803 \pm 0.0011$ (Bedding et al. (2007) using high precision asteroseismology) and $T_{\text {eff }}(K)=5872 \pm 44, R\left(\mathrm{R}_{\odot}=1.814 \pm 0.017\right), \log$ $g=3.952 \pm 0.005 L\left(\mathrm{~L}_{\odot}\right)=3.51 \pm 0.09$ and mass $M\left(\mathrm{M}_{\odot}\right)=1.07 \pm 0.03$ (North et al. (2007) using interferometry).

This, in addition to their luminosity-temperature relation which reflects their positions on the HR diagram and the 
evolutionary tracks (see Figure 4), their absolute magnitudes which are brighter than those of MS stars of the same temperatures, and their densities, leads us to conclude that both components are subgiant stars.

The age of the system was established from the evolutionary tracks as almost $2 \mathrm{~Gy}$. The similarity between the two components leads us to adopt the fragmentation process for the formation of the system, since it is the most likely mechanism in this case (for more discussion, see Bonnell 2001; Fabian, Pringle, \& Rees 1975; Binney \& Tremaine 1987).

\section{CONCLUSIONS}

The VCBS HD25811 is analysed using a modified version of the complex method which was first advised by Al-Wardat (2002a, 2007).

The modification in the method includes the dynamical analysis of the relative orbit of the system (which is very helpful in the determination of the masses of the individual components of the system) and consequently their spectral types, by combining them with the atmospheric modelling input parameters.

The physical and geometrical parameters of the system's components are estimated depending on the best fit between the observational and synthetic SEDs, built using the atmospheric modelling of the individual components and the system's orbital elements.

Both components of the system are concluded to be F2 IV for the primary and F1 IV for the secondary.

The entire and individual $U B V R$ Johnson-Cousins, $u v b y$ Strömgren, and $B V$ Tycho synthetic magnitudes and colours of the system are calculated.

Finally, fragmentation was proposed as the most likely process for the formation and evolution of the system.

\section{ACKNOWLEDGEMENTS}

This work made use of the Fourth Interferometric Catalogue, SIMBAD database, and CHORIZOS code of photometric and spectrophotometric data analysis (http://www.stsci.edu/jmaiz/software/ chorizos/chorizos.html). The authors thank Miss. Kawther Al-Waqfi for her help in some calculations and Mrs. Donna Keeley for the language editing.

\section{REFERENCES}

Al-Wardat, M. A. 2002a, BSAO, 53, 51

Al-Wardat, M. A. 2002b, BSAO, 53, 58

Al-Wardat, M. A. 2003, Phd dissertation, Special Astrophysical Observatory, RAN, Nizhnij Arkhyz, Zelenchukskiy region, 369167 RUSSIA, unpublished

Al-Wardat, M. A. 2007, AN, 328, 63

Al-Wardat, M. A. 2008, AstBu, 63, 361

Al-Wardat, M. A. 2009, AN, 330, 385

Al-Wardat, M. 2012, PASA, 29, 523

Al-Wardat, M. A., \& Widyan, H. 2009, AstBu, 64, 365

Bagnuolo, Jr, W. G., Mason, B. D., Barry, D. J., Hartkopf, W. I., \& McAlister, H. A. 1992, AJ, 103, 1399
Balega, I. I., \& Balega, Y. Y. 1987, PAZh, 13, 508

Balega, I. I., Balega, Y. Y., Belkin, I. N., Maximov, A. F., Orlov, V. G., Pluzhnik, E. A., Shkhagosheva, Z. U., \& Vasyuk, V. A. 1994, A\&AS, 105, 503

Balega, I. I., Balega, Y. Y., Hofmann, K.-H., Maksimov, A. F., Pluzhnik, E. A., Schertl, D., Shkhagosheva, Z. U., \& Weigelt, G. 2002, A\&A, 385, 87

Balega, I. I., Balega, Y. Y., Hofmann, K.-H., \& Weigelt, G. 2001, AstL, 27, 95

Balega, I. I., Balega, A. F., Maksimov, E. V., Malogolovets, E. A., Pluzhnik, E. A., \& Shkhagosheva, Z. U. 2006, BSAO, 59, 20

Balega, I. I., Balega, Y. Y., Maksimov, A. F., Malogolovets, E. V., Rastegaev, D. A., Shkhagosheva, Z. U., \& Weigelt, G. 2007, AstBu, 62, 339

Balega, I., Balega, Y. Y., Maksimov, A. F., Pluzhnik, E. A., Schertl, D., Shkhagosheva, Z. U., \& Weigelt, G. 2004, A\&A, 422, 627

Balega, I. I., Balega, Y. Y., \& Vasyuk, V. A. 1989, AISAO, 28, 107

Bedding, T. R., et al. 2007, ApJ, 663, 1315

Binney, J., \& Tremaine, S. 1987, in Galactic Dynamics, ed. J. Binney \& S. Tremaine, Princeton University Press, Princeton, NJ1987, 5

Bonnell, I. A. 2001, in IAU Symposium, Vol. 200; The Formation of Binary Stars, ed. H. Zinnecker \& R. Mathieu, International Astronomical Union, Paris, France, 23-32

Dravins, D., Lindegren, L., \& Vandenberg, D. A. 1998, A\&A, 330, 1077

ESA. 1997, The Hipparcos and Tycho Catalogues (ESA), VizieR Online Data Catalog, 1239, 0

Evans, D. S., Edwards, D. A., Frueh, M., McWilliam, A., \& Sandmann, W. H. 1985, AJ, 90, 2360

Fabian, A. C., Pringle, J. E., \& Rees, M. J. 1975, MNRAS, 172, $15 \mathrm{P}$

Fernandes, J., \& Monteiro, M. J. P. F. G. 2003, A\&A, 399, 243

Girardi, L., Bressan, A., Bertelli, G., \& Chiosi, C. 2000, A\&AS, 141,371

Gray, D. F. 2005, in The Observation and Analysis of Stellar Photospheres, ed. D. F. Gray, 3rd edn. Cambridge University Press, Cambridge, 505-508

Hartkopf, W. I., McAlister, H. A., \& Franz, O. G. 1992, AJ, 104, 810

Høg, E., et al. 2000, A\&A, 355, L27

Kurucz, R. 1994, Solar Abundance Model Atmospheres for 0,1,2,4,8 $\mathrm{km} \mathrm{s}^{-1}$. Kurucz CD-ROM No. 19 (Cambridge, MA: Smithsonian Astrophysical Observatory)

Lang, K. R. 1992, in Astrophysical Data I. Planets and Stars., ed. K. R. Lang. New York: Springer-Verlag, 133-139

Maíz Apellániz, J. 2006, AJ, 131, 1184

Maíz, Apellániz, J. 2007, in Astronomical Society of the Pacific Conference Series, ed. C. Sterken (Vol. 364; The Future of Photometric, Spectrophotometric and Polarimetric Standardization), San Francisco: Astronomical Society of the Pacific 227236

McAlister, H., Hartkopf, W. I., \& Franz, O. G. 1990, AJ, 99, 965

McAlister, H. A., Hartkopf, W. I., Hutter, D. J., \& Franz, O. G. 1987, AJ, 93, 688

McAlister, H. A., Hartkopf, W. I., Sowell, J. R., Dombrowski, E. G., \& Franz, O. G. 1989, AJ, 97, 510

North, J. R., et al. 2007, MNRAS, 380, L80

Schmidtke, P. C., \& Africano, J. L. 1984, AJ, 89, 1371

Straizys, V., \& Kuriliene, G. 1981, Ap\&SS, 80, 353 